\title{
BMJ Open Population Registry of Esophageal and Stomach Tumours in Ontario (PRESTO): protocol for a multicentre clinical and pathological database including 25 000 patients
}

To cite: Gupta V, Levy J, AllenAyodabo C, et al. Population Registry of Esophageal and Stomach Tumours in Ontario (PRESTO): protocol for a multicentre clinical and pathological database including 25000 patients. BMJ Open 2020;10:e032729. doi:10.1136/ bmjopen-2019-032729

- Prepublication history for this paper is available online. To view these files, please visit the journal online (http://dx. doi org/10.1136/bmjopen-2019032729).

Received 05 July 2019 Revised 06 February 2020 Accepted 09 April 2020
Check for updates

(C) Author(s) (or their employer(s)) 2020. Re-use permitted under CC BY-NC. No commercial re-use. See rights and permissions. Published by BMJ.

For numbered affiliations see end of article.

Correspondence to Dr Natalie G Coburn;

Natalie.Coburn@sunnybrook.ca

\section{ABSTRACT}

Introduction Oesophagogastric cancers carry a high mortality, economic burden and rising incidence. There is a need to monitor and improve care for this disease. Pathologic information is a cornerstone of cancer diagnosis, treatment and prognosis. Few populationbased studies combine pathology information and clinical outcomes. The objective of this study is to develop a clinical and pathological database of oesophagogastric cancers to study practice patterns, resource utilisation and clinical outcomes.

Methods and analysis The Population Registry of Esophageal and Stomach Tumours in Ontario (PRESTO) will include all patients with oesophagogastric cancer diagnosed from 2002 onwards within the province of Ontario. We estimate that the sample over the first 14 years of the study will include 26000 patients. Pathologic information from diagnostic procedures, endomucosal resection specimens and surgical resection specimens is being abstracted into a purpose-built database. Pathology information will be linked to administrative data, which capture baseline demographics, patient-reported symptoms, physician billings, hospital visits, hospital characteristics, geography and vital statistics. The registry will be updated prospectively.

Ethics and dissemination Ethics approval for this study was obtained from the Sunnybrook Health Sciences Centre Research Ethics Board. The PRESTO database will enable the study of oesophagogastric cancer in Ontario under six themes of inquiry: treatment, surgical outcomes, pathology, survival, health system and resource utilisation and cost. This information will be a valuable addition to the global efforts to understand ways to optimise care for these diseases.

\section{INTRODUCTION}

Improving care for oesophagogastric cancer is a key priority for clinicians and researchers as the prognosis for these malignancies remains dismal. ${ }^{1-3}$ Management requires multidisciplinary, specialised care, ${ }^{4-9}$ predisposing the
Strengths and limitations of this study

- The Population Registry of Esophageal and Stomach Tumours in Ontario (PRESTO) database will have near-complete case capture of oesophagogastric cancers diagnosed from a population of 14 million residents in Ontario, Canada.

- This is a population-based database with granular pathology data, reflecting real-world practice and outcomes and will be more generalisable.

- PRESTO will be the first clinical and pathological database for oesophagogastric cancer from North America and maximises the amount of information available.

- Data from clinical notes or radiology reports are not available, so cancer recurrence cannot be determined, and metastases can only be confirmed if biopsied

care for these malignancies to be inconsistent. ${ }^{10-14}$ Those suffering from oesophagogastric cancers experience a high degree of morbidity and mortality and the economic burden is felt on a personal and health system level. ${ }^{15-17}$

Current population-based studies in oesophagogastric cancer capture large, representative samples through administrative data. ${ }^{18-24}$ This improves generalisability, represents real-world care, offers research efficiency in time and money and can allow for greater longitudinal follow-up. However, population-based studies do not always contain pathology data, which are important for understanding disease characteristics, treatment response and surgical quality. ${ }^{25}$ Pathology data for oesophagogastric cancers are not part of Ontario's health administrative data holdings; linkage of administrative 
data sets with population-level pathology data would serve multiple purposes, including healthcare research and quality control.

The objective of the Population Registry of Esophageal and Stomach Tumours in Ontario (PRESTO) is to establish a population-based clinical and pathological database of all patients with oesophagogastric cancer to study practice patterns, resource utilisation and clinical outcomes. In this report, we describe the database development, population and implications for future work.

\section{METHODS}

\section{Study setting}

Ontario has a public, universal, single-payer health system serving its population of 13.9 million residents. Clinical, demographic and billing administrative data are routinely collected and held at Institute for Clinical Evaluative Sciences (ICES, which is a legally prescribed repository of patient information.

\section{Study population}

The PRESTO database will include all adult patients with oesophagogastric cancers (ICD-O-3 codes C15-C16, resected and non-resected) of any histology, diagnosed from 2002 onwards in Ontario, Canada. Patients will be identified through the Ontario Cancer Registry (OCR), which captures index cancer diagnoses through mandatory reporting. Patients less than 18 years of age, those not eligible for government health insurance, and those who died before the date of diagnosis will be excluded. We estimate that the samples over the first 14 years of the study will include 26000 patients. The PRESTO database will be updated biennially. The data will be held securely at ICES and made available to coinvestigators; it will not be available to researchers outside of ICES due to data privacy laws in Ontario.

\section{Data sources}

The PRESTO database will be derived from 17 administrative data sets and will include adults with oesophagogastric cancer identified through the OCR (table 1). OCR is a passive, provincial registry capturing $96 \%$ of incident cancer diagnoses in Ontario. ${ }^{26} 27$ The data sets will be linked through deterministic algorithms at ICES, using an encrypted number assigned to each patient treated through public health insurance. Pathology reports will be requested from the OCR based on the inclusion criteria, securely transferred to ICES and abstracted for pathology data. Reports will be abstracted for date of diagnosis, histology, grade, molecular characteristics, lymphovascular invasion status, perineural invasion status, tumour site, tumour size, treatment response, margin status, lymph node involvement, extra-nodal extensions and stage. Resection specimen pathology reports will be further queried for quantification and location of lymph node involvement, local invasion, evidence of metastatic disease and margin status.
Physician billing data (Ontario Health Insurance Plan (OHIP)) will be used to identify patients undergoing primary tumour resection (endomucosal and surgical resection), chemotherapy and radiotherapy and physician case volumes. Chemotherapy and radiation therapy visits will also be captured through a cancer centre treatment database (Activity Level Reporting). The inpatient and outpatient hospitalisation record data sets (Canadian Institute for Health Information and National Ambulatory Care Reporting System) will be used to determine diagnoses, hospital and intensive care unit length of stay, emergency department visits, readmissions, clinic visits, complications and interventions. Patient medications will be recorded from drug formulary registries which capture prescriptions for patients over the age of 65 (Ontario Drug Benefit) and new injectable cancer medications (New Drug Funding Program). Details on home-care services, rehabilitation and long-term or complex care will be available from the respective data sets (HomeCare Database, NationalRehabilitation Reporting System and ContinuingCare Reporting System). Patient-reported symptom scores will be available for a subset of patients attending cancer-centre outpatient visits (Symptom Management Reporting Database). Additional demographic data and sources will include immigration status (The Immigration, Refugees and Citizenship Canada Permanent Resident), socioeconomic status (Ontario Marginalization Index), place of residence and vital status (Registered Persons Database). Physician-level characteristics including age, sex, years in practice and fellowship training will be derived from provider databases (ICES Physician Database and Corporate Provider Database).

\section{Pathology data collection instrument}

A committee with representation from gastrointestinal pathology, surgical oncology, medical oncology, radiation oncology and clinical epidemiology has developed a standardised data collection instrument for pathology reports based on the American Joint Committee on Cancer (AJCC) guidelines for variables to include in a cancer registry ${ }^{28}$ and the College of American Pathologists' (CAP) published protocols for examining surgical specimens. ${ }^{29}{ }^{30}$ A Microsoft Access database with a userfriendly interface has been established based on the data collection instrument. The interface is designed with a permanently visible header capturing demographic information, tumour location, histology and the procedure for which the pathology report is generated. Types of procedures that will be captured include pre-treatment and surveillance endoscopic biopsies, endomucosal resection specimens, surgical resection specimens and biopsies of distant metastases. Abstraction will be categorised into the following three sections: biopsy specimen, surgical specimen and comments. The permanent header and histologic, cytopathologic and molecular information will be recorded for all reports. Surgical specimens reports will also be evaluated for lymph node or metastatic involvement, tumour response, surgical margin status, 
Table 1 Data sources for the PRESTO study

\section{Database}

Ontario Cancer Registry (OCR)

The Immigration, Refugees and Citizenship Canada Permanent Resident

Ontario Marginalization Index

Registered Persons Database

Pathology Report Data

Ontario Health Insurance Plan Database

Cancer Activity Level Reporting

Canadian Institute for Health Information Discharge Abstract Database and Same Day Surgery Database

National Ambulatory Care Reporting System

Ontario Drug Benefit and New Drug Funding Program

Home Care Database

National Rehabilitation Reporting System

Continuing Care Reporting System

\section{Symptom Management Reporting Database}

\section{Corporate Provider Database and ICES Physician Database}

\section{Information}

OCR is a passive, provincial registry that captures $96 \%$ of cancer diagnoses in Ontario. ${ }^{26} 27$ Created using data from hospital and regional cancer centre records, surgical pathology reports and Ontario death certificates.

National database of immigration records for individuals who have immigrated to Canada.

Specialised database using census data to profile relative area-level marginalisation (socioeconomic information) at various geographic levels in Ontario.

Derived from all administrative data sources and provides demographic data, vital status and details such as date of last contact with the healthcare system.

Pathology data abstracted by physicians from oesophagogastric cancer pathology reports identified through the OCR.

Inpatient and outpatient healthcare provider billing data based on submitted claims for remuneration.

Data set describing patient-level radiation and systemic therapy services and outpatient oncology clinic visits.

Patient and facility-level information from hospital admissions and outpatient surgical procedures; mandatory reporting system for all institutions in Canada.

Data from all hospital-based and community-based ambulatory care, including day surgery, outpatient clinics, cancer centre visits and emergency department visits.

Information on medication and chemotherapy administration for patients above the age of 65 and medication covered by provincial government insurance.

Captures all home care services provided or coordinated by Local Health Integration Networks, including nursing, personal care and paramedical support services.

Data from inpatient rehabilitation services including episode details and level of assistance required; mandatory reporting from all facilities with designated adult inpatient rehabilitation beds.

Contains demographic, clinical, functional and resource utilisation information from hospital-based care services for patients needing extended care, chronic care or complex continuing care, or from long-term care homes.

Contains patient-reported symptom scores for patients receiving oncologic care at a regional cancer centre.

Details on care providers, including speciality, area of practice, patient volume and year of graduation.

ICES, Institute for Clinical Evaluative Sciences; PRESTO, Population Registry of Esophageal and Stomach Tumours in Ontario.

local invasion and staging. A data dictionary has been developed as a reference standard for data abstraction and training.

\section{Pathology report abstraction}

To ensure detailed and reliable interpretation of the pathology reports, two medical doctors with graduatelevel research training (CA-A and EA) have been recruited to perform the data abstraction. Both abstractors have been trained by the principal investigator and co-investigators (NGC, VG, YJ and JL) on oesophagogastric cancer, relevant pathology data, key resources including the AJCC staging ${ }^{31}$ and CAP protocols. ${ }^{29} 30$ The data collection instrument has been tested for feasibility and clarity. Regular meetings will be held with the abstractors to review any questions, clarify abstraction protocols and provide advice or resources for unclear reports. Full committee data quality review will be performed as part of data cleaning. This included checks for plausibility, consistency and accuracy using clinical expertise, published literature and the abstraction manual. 
Table 2 Inter-rater reliability results for pathology data abstraction

\begin{tabular}{|c|c|}
\hline Variable & Per cent agreement \\
\hline $\begin{array}{l}\text { Date of pathology } \\
\text { report }\end{array}$ & 81.1 \\
\hline Type of procedure & 93.3 \\
\hline Histologic type & 87.8 \\
\hline Tumour location* & (97.8-99.6) \\
\hline Tumour grade & 86.3 \\
\hline $\begin{array}{l}\text { Number of positive } \\
\text { nodes }\end{array}$ & 94.0 \\
\hline $\begin{array}{l}\text { Location of positive } \\
\text { lymph nodes * }\end{array}$ & (99.5-99.8) \\
\hline Final margin* & $(95.8-98.8)$ \\
\hline $\begin{array}{l}\text { Staging system } \\
\text { (gastric or } \\
\text { oesophagus) }\end{array}$ & 98.0 \\
\hline
\end{tabular}

AJCC version (sixth or 97.5

seventh)

\begin{tabular}{lll} 
T stage $^{*}$ & 99.3 & $(98.9-99.5)$ \\
N stage* $^{*}$ & 99.5 & $(99.1-99.5)$ \\
\hline
\end{tabular}

${ }^{*}$ Median and IQR are presented where multiple variables were used to capture the datapoint.

AJCC, American Joint Committee on Cancer.

During our data quality check, we found that some patients are missing a pathology report in OCR, even when they had a diagnosis record in OCR and a surgical resection record in OHIP. We have fed this information back to the OCR, and future updates to PRESTO will include these pathology reports if they are identified.

\section{Inter-rater reliability}

Preliminary data abstraction of four hundred and two pathology reports was performed by two abstractors (CA-A and EA) to test the abstraction interface and determine inter-rater reliability. We calculated per cent agreement on 12 key variables determined a priori: date of pathology report, type of procedure, histologic type, tumour location, tumour grade, number of positive lymph nodes, final margin (proximal, distal and radial), tumour, node and metastases (TNM) staging method used (oesophagus or gastric), AJCC version used (sixth or seventh), T stage, $\mathrm{N}$ stage and location of lymph node metastases. All reports with any discrepant coding were reviewed individually and resolved by two coinvestigators (VG and JL) with both abstractors. This opportunity served as further training on abstraction protocols. Per cent concordance was greater than $95 \%$ for the majority of variables, indicating reliable capture of important variables (table 2). Date of pathology report had the lowest per cent agreement at $81 \%$; further investigation revealed this was a misunderstanding between date of pathology report generation and date of specimen collection. This was clarified and the variable was reabstracted with the correct definition, yielding $100 \%$ agreement.

\section{Exposures and outcomes}

The PRESTO database is a platform for a variety of investigations into oesophagogastric cancer care and outcomes. Exposures and outcomes will be defined for each individual study according to its research questions. Examples of key exposures to be investigated include receipt of surgery, receipt of chemoradiotherapy, surgeon-level characteristics such as training and hospital-level characteristics such as volume. Example outcomes of interest include hospital length of stay, surgical quality such as tumour margins and lymph node yield, readmission, mortality, complications and survival. These will be detailed in substudy manuscripts.

\section{Data safety}

ICES is a non-profit research organisation established under the sponsorship of the Ontario Ministry of Health and Long-Term Care and the Ontario Medical Association. It is a prescribed entity under Ontario's Personal Health Information Protection Act. All passive data sets held at ICES are subjected to ICES policies, practices and procedures for privacy protection and data security. Pathology report data will be entered directly into a Microsoft Access database within the secure ICES Abstractor Workroom and data environment.

\section{Patient and public involvement}

Patients and patient advocacy groups are being engaged to determine research priorities from the PRESTO database. They are providing insight into research questions, outcomes of interest, and the impact or relevance of proposed studies. We plan to collaborate with these partners to facilitate knowledge translation and to determine policy implications.

\section{Ethics and dissemination}

Ethics approval for this study was obtained from the Sunnybrook Health Sciences Centre Research Ethics Board. Results from the PRESTO database will be shared locally with key health policy and government leaders in Ontario, and internationally through peer-reviewed publications, to contribute to the global efforts to understand ways to optimise care for oesophagogastric cancer.

\section{DISCUSSION}

We are developing the PRESTO database to enable a comprehensive assessment of oesophagogastric cancer care. This will be a rich resource for the gastrooesophageal cancer community as it provides the opportunity to study a wide breadth and depth of questions in a real-world setting, on a population level.

\section{Research themes and opportunities}

Our research programme is organised into six themes of inquiry: treatment, surgical outcomes, pathology, 


\section{Research themes}

\begin{tabular}{|c|c|c|c|c|c|}
\hline Treatment & Surgical outcomes & Pathology & Survival & Health system & $\begin{array}{l}\text { Resource } \\
\text { utilisation and } \\
\text { cost }\end{array}$ \\
\hline $\begin{array}{l}\text { Treatment patterns, } \\
\text { for example, rates } \\
\text { of treatment with } \\
\text { multimodal therapy } \\
\text { Regional variation } \\
\text { in practice patterns } \\
\text { Treatment } \\
\text { effectiveness, } \\
\text { for example, } \\
\text { preoperative CT } \\
\text { versus CRT }\end{array}$ & $\begin{array}{l}\text { Risk factors, causes } \\
\text { and characteristics } \\
\text { of readmission } \\
\text { Reintervention } \\
\text { Mortality } \\
\text { Postoperative } \\
\text { complications } \\
\text { Hospital and ICU } \\
\text { length of stay }\end{array}$ & $\begin{array}{l}\text { Pathological results } \\
\text { of preoperative } \\
\text { treatment for } \\
\text { example, rates } \\
\text { and predictors of } \\
\text { complete response } \\
\text { Prognostic } \\
\text { importance of } \\
\text { pathology data } \\
\text { Quality of } \\
\text { pathology reporting }\end{array}$ & $\begin{array}{l}\text { Population-level } \\
\text { survival } \\
\text { Predictors of survival } \\
\text { Developing a } \\
\text { prediction tool } \\
\text { (nomogram) for } \\
\text { survival }\end{array}$ & $\begin{array}{l}\text { Effect of } \\
\text { centralised surgery } \\
\text { on outcomes } \\
\text { Uptake of clinical } \\
\text { trial data } \\
\text { Organisation and } \\
\text { delivery of care }\end{array}$ & $\begin{array}{l}\text { Cost of care } \\
\text { (overall) and } \\
\text { specific therapies } \\
\text { Economic } \\
\text { evaluations and } \\
\text { cost-effective } \\
\text { analyses }\end{array}$ \\
\hline
\end{tabular}

CRT, chemoradiotherapy; CT, chemotherapy; ICU, intensive care unit.

survival, health system and resource utilisation and cost. Examples of studies under each theme are given in table 3. The PRESTO group invites collaborators with research questions to provide evidence where knowledge gaps exist. PRESTO also presents a valuable opportunity to validate the results of other studies. In particular, it can help meet the need to validate prognostic tools designed to make individualised predictions for patients in a clinical setting. For example, we found that 17 survival prognostication tools exist for oesophageal and junctional cancer, but many are not validated and the majority have been designed for squamous cell cancer alone. ${ }^{33}$ Validation in our cohort can increase the generalisability and utility of these tools beyond the setting in which they were developed.

\section{Unique addition of pathology data}

A significant component of the PRESTO database is pathology data. Pathology reports are not routinely available for research purposes, and their lack of availability in an electronic database is a barrier to mobilising pathology data. We will perform manual abstraction of all pathology reports available for oesophagogastric cancer patients into a purpose-built database, with capture of clinically meaningful variables. This will greatly enhance detail and quality in PRESTO data, but is resourceintense and may not be feasible for other research groups. One solution we feel would facilitate research is computer-based, synoptic pathology reporting, with electronic data transfer into a centralised pathology database. This database could be auto-populated based on pre-determined synoptic fields, removing the need for manual abstraction. While manual abstraction is necessary for PRESTO in the absence of such a computerised system in Ontario, future research teams may be able to take advantage of such efficiency by advocating for electronic, centralised, synoptic pathology data reporting within their jurisdictions.
PRESTO's strengths compared to other existing data sets The PRESTO database will be a unique addition to the existing oesophagogastric cancer databases in the world. First, in terms of cancer registries, PRESTO will have near-complete case capture, ${ }^{26}{ }^{27}$ compared with the US Surveillance, Epidemiology, and End Results registry ${ }^{34}$ and the National Cancer Data Base, ${ }^{35}$ which capture $34 \%$ and $70 \%$ of incident cancer diagnoses, respectively. Second, PRESTO will be more generalisable than databases derived from high-volume academic centres. Many academic centres maintain prospective databases, ${ }^{36-38}$ and some have been linked to create data sets such as the Worldwide Esophageal Cancer Collaboration ${ }^{39}$ and Esophagectomy Complications Consensus Group. ${ }^{40}$ These studies are valuable for their granularity and ability to prospectively capture necessary data. They provide benchmarking and inform standard of care, but often cannot be generalised to real-world practice. Third, PRESTO will have more extensive clinical and pathology information than other similar population-level databases from Sweden, ${ }^{41}$ Netherlands, ${ }^{42}$ Japan $^{43}$ and England, ${ }^{44}$ among others. They often have high case ascertainment rates and add some pathology data to clinical data, but no database provides a complete clinical and pathological picture of patients that would be needed to understand characteristics and account for relevant factors. Importantly, PRESTO includes patient-reported symptom scores, which are collected at each outpatient cancer centre visit in Ontario, and offers the ability to look at patients' health-related quality of life. PRESTO will be the first clinical and pathological database for oesophagogastric cancer from North America and maximises the amount of information available.

\section{Limitations}

The PRESTO database has some limitations to note. Data from clinical notes or radiology reports are not collected centrally in Ontario, and so are not available in PRESTO; 
as a result, cancer recurrence cannot be determined, and metastases can only be confirmed if biopsied. We have previously developed an algorithm to identify metastatic gastric cancer patients in the absence of staging data, using administrative data, which we plan to use in PRESTO. ${ }^{45}$ Administrative data codes capture procedures performed (eg, oesophagectomy and/or gastrectomy) and whether minimally invasive approaches were used (eg, laparoscopic and/or thoracoscopic approach), but do not contain details on type of resection (eg, transhiatal, transthoracic and so on). We can extract cancer stage from pathology reports for resected patients but will not have stage for unresected patients. Unmeasured confounders remain, including race, performance status and lifestyle characteristics such as diet, smoking or obesity. There is potential misclassification when defining variables of interest. For example, when defining curative versus palliative radiotherapy, we use information such as radiation dose, intent of therapy or administrative delivery codes to separate the two groups. When making such definitions, we employ validated or previously used algorithms as much as possible, but there may be imperfect classification since we are deriving this from secondary sources. Finally, when performing comparative effectiveness research, we recognise that selection bias will exist in retrospective data resulting from clinical decision-making.

\section{CONCLUSION}

In conclusion, the PRESTO database will enable the study of treatment patterns, geographic variation, resource utilisation and clinical outcomes for oesophagogastric cancer in a real-world setting using population-based data from Ontario. This information will be a valuable addition to the global efforts to understand ways to optimise care for this disease. The PRESTO group welcomes collaboration with interested researchers.

\section{Author affiliations}

${ }^{1}$ Department of Surgery and Institute of Health Policy, Management, and Evaluation, University of Toronto, Toronto, Ontario, Canada

${ }^{2}$ Evaluative Clinical Sciences, Sunnybrook Research Institute, Toronto, Ontario, Canada

${ }^{3}$ Analysis, Institute for Clinical Evaluative Sciences, Toronto, Ontario, Canada

${ }^{4}$ Community Health Sciences, University of Manitoba College of Medicine, Winnipeg, Ontario, Canada

${ }^{5}$ Division of General Surgery, Department of Surgery and Institute of Health Policy, Management, and Evaluation, Odette Cancer Centre, Toronto, Ontario, Canada

Twitter Vaibhav Gupta @VGupta_MD

Acknowledgements This study is supported by the Sherif and MaryLou Hanna Chair in Surgical Oncology at Sunnybrook Health Sciences Centre.

Collaborators PRESTO Study Investigators: Vaibhav Gupta, Division of General Surgery, Department of Surgery and Institute of Health Policy, Management, and Evaluation, University of Toronto, Canada. Jordan Levy, Division of General Surgery, Department of Surgery and Institute of Health Policy, Management, and Evaluation, University of Toronto, Canada. Catherine Allen-Ayodabo, Evaluative Clinical Sciences, Sunnybrook Research Institute, Toronto, Canada. Elmira Amirazodi, Evaluative Clinical Sciences, Sunnybrook Research Institute, Toronto, Canada. Yunni Jeong, Division of General Surgery, Department of Surgery and Institute of
Health Policy, Management, and Evaluation, University of Toronto, Canada. Laura E. Davis, Evaluative Clinical Sciences, Sunnybrook Research Institute, Toronto, Canada. Qing Li, MSc. Institute for Clinical Evaluative Sciences, Toronto, Canada. James R. Conner, Department of Laboratory Medicine and Pathobiology, University of Toronto; Mount Sinai Hospital, Toronto, Canada. Eugene Hsieh, Department of Laboratory Medicine and Pathobiology, University of Toronto; Sunnybrook Health Sciences Centre, Toronto, Canada. Alyson L. Mahar, Manitoba Centre for Health Policy and Department of Community Health Sciences, University of Manitoba, Canada. Jolie Ringash, MD, MSc. Department of Radiation Oncology and Institute of Health Policy, Management, and Evaluation, University of Toronto, Canada. Elena Elimova, Division of Medical Oncology, Department of Medicine, University of Toronto; Princess Margaret Cancer Centre, Toronto, Canada. Rinku Sutradhar, PhD. Institute for Clinical Evaluative Sciences and Institute of Health Policy, Management, and Evaluation, University of Toronto, Canada. Biniam Kidane, Section of Thoracic Surgery, Department of Surgery, University of Manitoba. Gail E. Darling, Department of Surgery and Institute of Health Policy, Management, and Evaluation, University of Toronto; Toronto General Hospital/University Health Network, Toronto, Canada. Natalie G. Coburn, Division of General Surgery, Department of Surgery and Institute of Health Policy, Management, and Evaluation, University of Toronto; Sunnybrook Health Sciences Centre, Toronto, Canada.

Contributors VG and NGC designed the study with input from all authors. VG, CA-A, EA, JL, LED, ALM, QL and NGC acquired the data. QL analysed the data, and it was interpreted by VG, JL and NGC with input from all authors. VG and JL drafted the manuscript and all authors revised it critically. All authors and PRESTO investigators approved the final version for publication and are accountable for all aspects of the work.

Funding This study is supported by the Sherif and MaryLou Hanna Chair in Surgical Oncology at Sunnybrook Health Sciences Centre.

Competing interests NGC and GED receive salary support from Ontario HealthCancer Care Ontario.

Patient and public involvement Patients and/or the public were not involved in the design, or conduct, or reporting, or dissemination plans of this research.

Patient consent for publication Not required.

Provenance and peer review Not commissioned; externally peer reviewed.

Open access This is an open access article distributed in accordance with the Creative Commons Attribution Non Commercial (CC BY-NC 4.0) license, which permits others to distribute, remix, adapt, build upon this work non-commercially, and license their derivative works on different terms, provided the original work is properly cited, appropriate credit is given, any changes made indicated, and the use is non-commercial. See: http://creativecommons.org/licenses/by-nc/4.0/.

ORCID iD

Vaibhav Gupta http://orcid.org/0000-0003-4568-121X

\section{REFERENCES}

1 Howlader N, Noone A, Krapcho M, et al. SEER Cancer Statistics Review, 1975-2014. In: . 2017. Bethesda, MD: National Cancer Institute, 2017.

2 Canadian Cancer Society's Advisory Committee on Cancer Statistics. Canadian cancer statistics 2017. Toronto, Canada: Canadian Cancer Society, 2017.

3 Matsuda T, Saika K. The 5-year relative survival rate of stomach cancer in the USA, Europe and Japan. Jpn J Clin Oncol 2013;43:1157-8.

4 Smyth EC, Verheij M, Allum W, et al. Gastric cancer: ESMO clinical practice guidelines for diagnosis, treatment and follow-up. Ann Oncol 2016;27:v38-49.

5 Brar SS, Mahar AL, Helyer LK, et al. Processes of care in the multidisciplinary treatment of gastric cancer: results of a RAND/ UCLA expert panel. JAMA Surg 2014;149:18-25.

6 Ajani JA, D'Amico TA, Almhanna K, et al. Esophageal and esophagogastric junction cancers, version 1.2015. J Nat/ Compr Canc Netw 2015;13:194-227.

7 Ajani JA, D'Amico TA, Almhanna K, et al. Gastric cancer, version 3.2016, NCCN clinical practice guidelines in oncology. J Natl Compr Canc Netw 2016;14:1286-312.

8 Stahl M, Oliveira J, Group EGWEsophageal cancer: ESMO clinical recommendations for diagnosis, treatment and follow-up. Ann Oncol 2009;20 Suppl 4:iv32-3. 
9 Berry MF. Esophageal cancer: staging system and guidelines for staging and treatment. J Thorac Dis 2014;6 Suppl 3:S289-97.

10 Sherman KL, Merkow RP, Bilimoria KY, et al. Treatment trends and predictors of adjuvant and neoadjuvant therapy for gastric adenocarcinoma in the United States. Ann Surg Oncol 2013;20:362-70.

11 Coburn NG, Lourenco LG, Rossi SE, et al. Management of gastric cancer in Ontario. J Surg Oncol 2010;102:54-63.

12 Coburn NG, Swallow CJ, Kiss A, et al. Significant regional variation in adequacy of lymph node assessment and survival in gastric cancer. Cancer 2006;107:2143-51.

13 Mahar AL, El-Sedfy A, Dixon M, et al. Geographic variation in surgical practice patterns and outcomes for resected nonmetastatic gastric cancer in Ontario. Curr Oncol 2018;25:e436-e443.

14 Dikken JL, van Sandick JW, Allum WH, et al. Differences in outcomes of oesophageal and gastric cancer surgery across Europe. Br J Surg 2013;100:83-94.

15 De Oliveira C, Pataky R, Bremner KE, et al. Estimating the cost of cancer care in British Columbia and Ontario: a Canadian InterProvincial comparison. Healthc Policy 2017;12:95-108.

16 Ferlay J, Soerjomataram I, Dikshit R, et al. Cancer incidence and mortality worldwide: sources, methods and major patterns in GLOBOCAN 2012. Int J Cancer 2015;136:E359-86.

17 Yabroff KR, Lamont EB, Mariotto A, et al. Cost of care for elderly cancer patients in the United States. J Natl Cancer Inst 2008;100:630-41.

18 Dimick JB, Cattaneo SM, Lipsett PA, et al. Hospital volume is related to clinical and economic outcomes of esophageal resection in Maryland. Ann Thorac Surg 2001;72:334-40. discussion 9-41.

19 Chang AC, Ji H, Birkmeyer NJ, et al. Outcomes after transhiatal and transthoracic esophagectomy for cancer. Ann Thorac Surg 2008;85:424-9.

20 Peyre CG, Hagen JA, DeMeester SR, et al. The number of lymph nodes removed predicts survival in esophageal cancer: an international study on the impact of extent of surgical resection. Ann Surg 2008;248:190-7.

21 Wright CD, Kucharczuk JC, O'Brien SM, et al. Predictors of major morbidity and mortality after esophagectomy for esophageal cancer: a Society of thoracic surgeons General thoracic surgery database risk adjustment model. J Thorac Cardiovasc Surg 2009;137:587-96.

22 Tinmouth J, Green J, Ko Y-J, et al. A population-based analysis of esophageal and gastric cardia adenocarcinomas in Ontario, Canada: incidence, risk factors, and regional variation. J Gastrointest Surg 2011;15:782-90.

23 Kurita N, Miyata H, Gotoh M, et al. Risk model for distal gastrectomy when treating gastric cancer on the basis of data from 33,917 Japanese patients collected using a nationwide web-based data entry system. Ann Surg 2015;262:295-303.

24 Batista Rodríguez G, Balla A, Fernández-Ananín S, et al. The era of the large databases: outcomes after gastroesophageal surgery according to NSQIP, NIS, and NCDB databases. systematic literature review. Surg Innov 2018;25:400-12.

25 In H, Bilimoria KY, Stewart AK, et al. Cancer recurrence: an important but missing variable in National cancer registries. Ann Surg Oncol 2014;21:1520-9.

26 Robles SC, Marrett LD, Clarke EA, et al. An application of capturerecapture methods to the estimation of completeness of cancer registration. J Clin Epidemiol 1988;41:495-501.
27 Clarke EA, Marrett LD, Kreiger N. Cancer registration in ontario: a computer approach. In: Jensen OM, Parkin DM, MacLennan R, et al, eds. Cancer registration principles and methods. Pub No. 95 ed. Lyon, France: IARC, 1991: 246-57p.

28 Rice TW, Patil DT, Blackstone EH. 8Th edition AJCC/UICC staging of cancers of the esophagus and esophagogastric junction: application to clinical practice. Ann Cardiothorac Surg 2017;6:119-30.

29 Shi C, Berlin J, Branton PA, et al. Protocol for the examination of specimens from patients with carcinoma of the esophagus, version 4.0.0.0. Northfield, IL: College of American Pathologists, 2017.

30 Shi C, Berlin J, Branton PA, et al. Protocol for the examination of specimens from patients with carcinoma of the stomach, version 4.0.0.0. Northfield, IL: College of American Pathologists, 2017.

31 Washington K. 7Th edition of the AJCC cancer staging manual: stomach. Ann Surg Oncol 2010;17:3077-9.

32 Rice TW, Blackstone EH, Rusch VW. 7Th edition of the AJCC cancer staging manual: esophagus and esophagogastric junction. Ann Surg Oncol 2010;17:1721-4.

33 Gupta V, Coburn N, Kidane B, et al. Survival prediction tools for esophageal and gastroesophageal junction cancer: a systematic review. J Thorac Cardiovasc Surg 2018;156:847-56.

34 National Cancer Institute. Overview of the Surveillence, epidemiology, and end results (SEER) program, 2017. Available: https://seer.cancer.gov/about/overview.html

35 American College of Surgeons. National cancer database, 2018. Available: https://www.facs.org/quality-programs/cancer/ncdb

36 Markar SR, Noordman BJ, Mackenzie H, et al. Multimodality treatment for esophageal adenocarcinoma: multi-center propensityscore matched study. Ann Oncol 2017;28:519-27.

37 Markar S, Gronnier C, Duhamel A, et al. Pattern of postoperative mortality after esophageal cancer resection according to center volume: results from a large European multicenter study. Ann Surg Oncol 2015;22:2615-23.

38 Anderegg MCJ, Lagarde SM, Jagadesham VP, et al. Prognostic significance of the location of lymph node metastases in patients with adenocarcinoma of the distal esophagus or gastroesophageal junction. Ann Surg 2016;264:847-53.

39 Rice TW, Chen L-Q, Hofstetter WL, et al. Worldwide esophageal cancer collaboration: pathologic staging data. Dis Esophagus 2016;29:724-33.

40 Low DE, Kuppusamy MK, Alderson D, et al. Benchmarking complications associated with esophagectomy. Ann Surg 2017.

41 Markar SR, Mackenzie H, Lagergren P, et al. Surgical proficiency gain and survival after esophagectomy for cancer. J Clin Oncol 2016;34:1528-36.

42 Busweiler LAD, Wijnhoven BPL, van Berge Henegouwen MI, et al. The Dutch upper Gi cancer audit: 2011-2014. Journal of Clinical Oncology 2016;34:309.

43 Nashimoto A, Akazawa K, Isobe Y, et al. Gastric cancer treated in 2002 in Japan: 2009 annual report of the JGCA nationwide registry. Gastric Cancer 2013;16:1-27.

44 Coupland VH, Lagergren J, Lüchtenborg M, et al. Hospital volume, proportion resected and mortality from oesophageal and gastric cancer: a population-based study in England, 2004-2008. Gut 2013;62:961-6.

45 Mahar AL, Jeong Y, Zagorski B, et al. Validating an algorithm to identify metastatic gastric cancer in the absence of routinely collected TNM staging data. BMC Health Serv Res 2018;18:309. 